\title{
Freßverhalten und Darminhaltsuntersuchungen bei Sipunculiden
}

\author{
M. D. WALTER \\ Institut für Hydrobiologie und Fischereiwissenschafl der Universität Hamburg; \\ Hamburg 50, Bundesrepublik Deutscbland
}

\begin{abstract}
Feeding and studies on the gut content in sipunculids. Three sipunculid species were caught in two different biotopes: Phascolosoma vulgare (DE BLAINvinLE) as well as Pbascolosoma elongatum (KEFERSTEIN) from a "silt"-bottom in Gullmarsfjord (Sweden) and Physcosoma granulatum (Keferstern) from the "cotalligène" near. Banyuls-sur-mer (Mediterranean Sea). Observations on feeding behavior showed that the uptake of food is of a "passively selective" type. Apparently aimlessly, the sipunculids draw in sediment with food from the surroundings, often leaving starlike traces. Examination of gut contents revealed changes in sediment structure in different gut parts. The changes correspond to the different morphological and physiological functions of the gut. The main meiofauna food of the three species, especially nematodes and crustaceans, dominates also in their biotopes. Comparison of the numbers of the meiofauna specimens in the three different parts of the gut showed the expected decrease of food animals. Such decrease applies to all meiofauna-elements; plantdebris seems to pass the intestines unaffected. In order to examine a possible correlation between food and sipunculid size, the latter were classified according to body lenght. Decrease in average nematode lenght is positively correlated to diminishing body size of sipunculids. The same holds for copepods. The food ingested also comprises other organic particles and probably dissolved organic substances.
\end{abstract}

\section{EINLEITUNG}

Untersuchungen über trophische Systeme im Benthos liegen bisher nur in geringem Umfang vor (MARE 1942, GERLACH 1969), da die Funktionen und Leistungen der einzelnen Arten in den Lebensgemeinschaften des Meeresbodens meist nicht oder nur unzureichend bekannt sind. Bei produktionsbiologischen Untersuchungen dieses Lebens-" raumes stellt sich die Frage, welche Rolle den Vertretern der Meiofauna als Nahrung der Makrofauna zukommt. So haben z. B. GerLach \& SCHrage (1969) nachgewiesen, daß Nematoden als Nahrung für Sandgarnelen dienen können.

In diesem Zusammenhang sind bisher die reinen Sedimentfresser unberücksichtigt geblieben, die das Sediment und nicht selektiv bestimmte Größenordnungen von Partikeln bevorzugen. Sie wurden von Jørgensen $(1966,1970)$ sowie von TÉTRY (1959) als "mictophagous feeders" bezeichnet. $\mathrm{Zu}$ ihnen zählen die Holothurien (Angaben und Untersuchungen zum Freßverhalten bei CuENot 1948, RHoAds \& Young 1971) und die Sipunculiden. 
Diese Arbeit beschäftigt sich mit der Meiofauna als Nahrung für Sipunculiden. Durch Darminhaltsuntersuchungen sollte geklärt werden, ob die Sipunculiden Meiofauna in den Darmtrakt aufnehmen und ob eine bestimmte Relation zwischen den Größen der Sipunculiden und ihren Nahrungsorganismen besteht.

\section{MATERIAL UND METHODEN}

Das Verhältnis von Nahrungsangebot zu Nahrungsaufnahme bezüglich der Meiofauna sollte durch Darminhaltsuntersuchungen an drei Sipunculiden festgestellt werden: Phascolosoma vulgare (DE BLAInville), Pbascolosoma elongatum (KeFERSTEIN) und Physcosoma granulatum (F. S. LEUCKART).

Phascolosoma vulgare und Phascolosoma elongatum wurden während eines Aufenthaltes an der Zoologischen Station Kristineberg in Schweden im September 1971 gesammelt. Der Fundort „Blackhall" liegt im Schärengebiet zwischen dem Gullmarsfjord und dem Skagerrak; das Terrain umfaßt etwa $800 \mathrm{qm}$. Die Tiere wurden in einer Tiefe von $25 \mathrm{~m}$ gedredged. Das Sediment kann als eine Mischung von Schill und Sand mit "silt" charakterisiert werden. Die Lebensgemeinschaft, in der die beiden PhascolosomaArten an diesem Fundort vorkommen, ist nach Molander (1928) die Brissopsis-chiajei +Rhodine-Assoziation. Nach LEPrÄKOsKI (persönliche Mitteilung) beträgt der Salzgehalt in diesem Gebiet 30-31\%, die Bodentemperatur lag während der Zeit der Aufsammlungen bei $13^{\circ} \mathrm{C}$. Ein Dredgefang von ca. 10 min erbrachte durchschnittich insgesamt 15-20 Individuen beider Arten.

Die Tiere wurden ohne Betäubung in $4 \%$ Formalin-Seewasser konserviert. Diese Art der Fixierung bewirkte zwar eine geringere Kontraktion der Tiere, jedoch konnten nur durch diese Methode der weitere Nahrungstransport im Darm und somit eine Verfälschung der Ergebnisse unterbunden werden. Um eine Abhängigkeit der aufgenommenen Nahrung von der Individuengröße der Sipunculiden zu erkennen, wurden sie in Größenklassen aufgeteilt (Tab. 1).

Bei allen untersuchten Arten wurden die konservierten Tiere dorsal geöffnet, der Darm freigelegt und die Darmschlingen durch „Entwickeln“ voneinander getrennt. Die äußerlich gut voneinander zu unterscheidenden Darmabschnitte (Tab. 2) wurden einzeln auf ihren Gehalt an Meiofauna untersucht. Dazu wurde das aufgenommene Sediment mit Bengalrosa + Phenol (THiel 1966) angefärbt und ausgesucht.

Pbyscosoma granulatum stammt aus dem Corralligène in der Nähe der Biologischen Station Banyuls-sur-mer. Physcosoma granulatum ist ein sedentärer Endolith,

Tabelle 1

Größe und Anzahl der drei untersuchten Sipunculiden-Arten

\begin{tabular}{|cccc|}
\hline Größenklassen & $\begin{array}{c}\text { Phascolosoma } \\
\text { valgare }\end{array}$ & $\begin{array}{c}\text { Phascolosoma } \\
\text { elongatum }\end{array}$ & $\begin{array}{c}\text { Physcosoma } \\
\text { granulatum }\end{array}$ \\
\hline I $(1,0-2,4 \mathrm{~cm})$ & 23 & -1 & 26 \\
II $(2,5-4,9 \mathrm{~cm})$ & 31 & 16 & - \\
III $(5,0-7,4 \mathrm{~cm})$ & 21 & 8 & - \\
IV $(7,5-9,9 \mathrm{~cm})$ & - & & 16 \\
\hline
\end{tabular}


der strikt im Inneren der Verkalkungen lebt. Als Nahrung werden kleinere Endolithen und Schlammbewohner aufgenommen, die in den Sedimentationshöhlen des Corralligène leben. Die bearbeiteten Exemplare wurden aus Corralligène-Stücken, die aus etwa $25 \mathrm{~m}$ Tiefe gesammelt worden waren, herausgeschlagen. Sie wurden ebenfalls in

Tabelle 2

Morphologische und physiologische Kennzeichen des Darmes (nach TÉTry 1959) und Struktur des darin enthaltenen Sedimentes der drei untersuchten Sipunculiden-Arten

\begin{tabular}{|llll|}
\hline Darmabschnitte & $\begin{array}{c}\text { Morphologische } \\
\text { Kennzeichen }\end{array}$ & $\begin{array}{l}\text { Physiologische } \\
\text { Kennzeichen }\end{array}$ & \multicolumn{1}{c|}{$\begin{array}{c}\text { Struktur des } \\
\text { Sedimentes }\end{array}$} \\
\hline Abschnitt 1 & $\begin{array}{l}\text { Längsgestreites, } \\
\text { dickes Hautepithel }\end{array}$ & $\begin{array}{l}\text { Mechanischer } \\
\text { Nahrungstransport }\end{array}$ & Kurze Stäbchen \\
Abschnitt 2 & $\begin{array}{l}\text { Glattes, durchsichtiges } \\
\text { Epithel }\end{array}$ & $\begin{array}{l}\text { Resorption, Vor- } \\
\text { kommen von Phos- } \\
\text { phatasen, Lipasen } \\
\text { Mechanischer } \\
\text { Nahrungstransport }\end{array}$ & $\begin{array}{l}\text { Lange Stäbchen, locker } \\
\text { gepackt }\end{array}$ \\
Abschnitt 3 & Glattes Epithel & $\begin{array}{l}\text { Fest gepacktes, kom- } \\
\text { paktes Sediment }\end{array}$ \\
\hline
\end{tabular}

$4 \%$ Formalin-Seewasser konserviert. Die 26 Exemplare entsprechen der Größenklasse I der Pbascolosoma-Arten und wurden in drei Untergruppen eingeteilt (Tab. 1). Die Bearbeitung erfolgte in der gleichen Weise wie bei Phascolosoma vulgare und Phascolosoma elongatum.

Um vergleichende Untersuchungen über das vorhandene Nahrungsangebot durchführen $\mathrm{zu}$ können, wurden Bodenproben aus dem Gebiet „Blackhall" mit einem VanVeen-Greifer entnommen. Mit dem „Meiostecher“ (THIEL 1966) wurden Teilproben von $25 \mathrm{~cm}^{2}$ Sedimentoberfläche und $2 \mathrm{~cm}$ Einstichtiefe ausgewertet. Diese wurden jeweils in vier $1 / 2 \mathrm{~cm}$ starke Schichten aufgeteilt und mit $4 \%$ Formalin-Seewasser konserviert.

Vom Lebensraum von Physcosoma granulatum stand nur ein kleines Stück Corralligène zur Verfügung, in das sich ein Tier eingezwängt hatte. Um einen Einblick in das Nahrungsangebot zu erhalten, wurde dieses Bruchstück mit einem scharfen Wasserstrahl ausgespült. (Ausführliche Angaben zur Biocoenose finden sich bei LAUBIER 1966.)

Die Meiofauna wurde wie in den Darmabschnitten der Sipunculiden mit Bengalrosa + Phenol angefärbt und ausgesucht.

\section{ERGEBNISSE}

Beobachtungen an den drei Sipunculiden-Arten im Laboratorium - sie konnten über 8 Wochen lang in natürlichem Sediment bei $15^{\circ} \mathrm{C}$ Raumtemperatur gehältert werden - zeigten, daß sie U-förmig im Schlamm liegen und ihre Nahrung mit dem Sediment aus ihrer direkten Umgebung aufnehmen. Sie tasten die Sedimentoberfläche mit ihrem Tentakelkranz $a b$, wobei häufig sternförmige Freßspuren entstehen. Beim Einziehen des Rüssels bleiben an dessen Papillen Sandkörner und Nahrungspartikel haften. 
Auf Grund dieser Beobachtungen und der Ausführungen über das Verhalten der Sipunculiden (Peebles \& Fox 1933, MacGinitie \& MacGinitie 1949, Hyman 1959), daß die Tiere von der Wasser-Sediment-Grenzfläche bzw. den oberen $1 / 2-1 \mathrm{~cm}$ des Sedimentes fressen, war anzunehmen, daß die Tiere auch Meiofauna als Nahrung aufnehmen.

Die Darminhaltsuntersuchungen haben bestätigt, daß die Sipunculiden mit dem Sediment auch Meiofauna (Nematoden, Copepoden, Nauplien, Polychaeten, Mollusken, Foraminiferen, Turbellarien (?), Bryozoenteile und Hydroidpolypen) fressen.

Die $\mathrm{Nem}$ a toden stellen zahlen- und volumenmäßig den größten Anteil am tierischen Material im Darm der Sipunculiden dar; so finden sich z. B. in einem Individuum von Phascolosoma vulgare der Größenklasse II insgesamt 41 Nematoden. Die größeren Individuen dieser Art enthalten im Vorderdarm durchschnittlich 3-5 Nematoden, im Mitteldarm 2 Nematoden. Phascolosoma elongatum und Physcosoma granulatum enthalten nur insgesamt bis zu 3 Nematoden pro Individuum. Die Nematoden wurden in Längenklassen eingeteilt, da vermutlich allein die Länge der Nematoden deren Aufnahme durch die Sipunculiden verhindern kann. Ein Vergleich der Längen von Sipunculiden und aufgenommenen Nematoden zeigt deutlid, daß die Länge der Nematoden im Darmtrakt parallel zur Abnahme der Größenklasse verläut (Tab. 3).

Tabelle 3

Durchschnittliche Länge der aufgenommenen Nematoden im Darm der Sipunculiden und die Anzahl der vermessenen Individuen (in Klammern)

\begin{tabular}{|cccc|}
\hline Größenklasse & $\begin{array}{c}\text { Phascolosoma } \\
\text { vulgare }\end{array}$ & $\begin{array}{c}\text { Phascolosoma } \\
\text { elongatum }\end{array}$ & $\begin{array}{c}\text { Physcosoma } \\
\text { granulatum }\end{array}$ \\
\hline I & $0,24 \mathrm{~mm}(2)$ & $0,35 \mathrm{~mm}(5)$ \\
II & $0,36 \mathrm{~mm}(256)$ & $0,23 \mathrm{~mm}(4)$ & \\
III & $0,50 \mathrm{~mm}(96)$ & $0,36 \mathrm{~mm}(15)$ & \\
IV & & $0,47 \mathrm{~mm}(5)$ & \\
\hline
\end{tabular}

Jeweils in der Größenklasse I-II ist nur eine geringe Anzahl von Nematoden festzustellen. Außerdem finden sich auch im Enddarm noch einzelne Individuen. Es muß daher angenommen werden, daß eine vollständige Verdauung, möglicherweise auf Grund des relativ resistenten Hautmuskelschlaudhes der Nematoden, nicht erfolgen kann.

Auffallend ist der Zustand der Nematoden im Darm: Sie sind flach zusammengepreßt, offensichtlich eine Folge der schon erwähnten Bildung der stäbchenförmigen Gebilde im Mitteldarm; hierbei handelt es sich um eine wohl rein mechanisch erfolgte Veränderung.

Die Crustaceen (Harpacticiden, Ostracoden, Nauplien) zeigen in ihrer Verteilung in den Größengruppen und im Verlauf durch den Darmtrakt der Sipunculiden eine ähnliche Verteilung wie die Nematoden. Nur die größere Art, Phascolosoma vulgare, enthält nennenswerte Mengen von Crustaceen, wobei allerdings ein sprunghaftes Absinken der Individuenzahlen im Darm zu beobachten ist; so sinkt beim Obergang von Größenklasse III in II die durchschnittliche Crustaceenzahl im Darm von 1 Individuum auf 0,3 ab. Phascolosoma elongatum kann wegen seiner geringen 
Größe kaum Crustaceen aufnehmen; bei Physcosoma granulatum entspricht das Fehlen (bis auf wenige Ausnahmen) der Crustaceen (1) der geringen Größe der Sipunculiden, aber offensichtlich auch (2) dem Mangel von Crustaceen im zur Verfügung stehenden Nahrungsangebot.

Die Verdauung der Crustaceen beginnt offensichtlich in derem Inneren, um dann zuletzt auf das Chitingehäuse (z. T. angegriffene Exuvien) gegen Ende des Mitteldarmes überzugreifen. Beweis dafür ist das Vorkommen leerer, d. h. nicht mehr anfärbbarer Exuvien und von Bruchstücken im Enddarm. Im umgebenden Sediment wurden sie nicht so häufig und regelmäßig wie im Darm gefunden; ein zufällig gehäuftes Vorkommen ist nicht zu erwarten.

Das Vorkommen von Moll u s k en im Darm von Sipunculiden beschränkt sich auf die größte Art, Phascolosoma vulgare. Es ist allerdings nicht zu entscheiden, ob es sich um leere Schalen gehandelt hat oder um Exemplare, die bei der Aufnahme noch lebten und als Nahrung genutzt werden konnten. Das gleiche gilt auch für die in allen Größenklassen gefundenen $F$ or a $\mathrm{m}$ in if ere $n$ und $\mathrm{B}$ r yoz o e $\mathrm{n}$-Teilen.

Die im Darm nachgewiesenen Diat omeen sind vorwiegend planktischer Herkunft: Sie entstammen also dem Detritus. Im Vergleich zu den Phascolosoma-Arten wurden von Pbyscosoma granulatum relativ viele Diatomeen aufgenommen. In allen drei Darmabschnitten waren etwa gleichviel Pflanzenfaserteile und -bruchstücke vorhanden und durch Bengalrosa gleichmäßig angefärbt. Eine Verdauung des pflanzlichen Materials erfolgt offensichtlich nicht. Das bezweifelt auch Goerke (1971a) nach seinen Untersuchungen an Nereis virens, in dessen Enddarm er ebenfalls noch Pflanzenteile fand.

Um die Möglichkeit selektiven Fressens prüfen zu können, wurden Sedimentproben aus den Biotopen der Sipunculiden auf ihren Meiofaunagehalt untersucht. Es ergaben sich etwas unterschiedliche Zahlen, die aber der allgemein auftretenden Variabilität (MCINTYRE 1969) entsprechen.

Das Sediment von "Bladkhall“, Fundort von Phascolosoma vulgare und Pbascolosoma elongatum, enthielt pro Teilprobe von $25 \mathrm{~cm}^{2}$ Sedimentoberfläche und $1 / 2 \mathrm{~cm}$ Schichttiefe rund 1000-1500 Nematoden, 100-150 Harpacticiden, 70-150 Nauplien sowie einzelne Polychaeten, Ostracoden, Halacariden, Echinoiden, Mollusken und Diatomeen in der Sedimentfraktion $<150 \mu \mathrm{m}$. Das für Physcosoma granulatum zur Verfügung stehende Nahrungsangebot aus einem Bruchstück Corralligène ließ sich nicht quantitativ erfassen. Es enthielt Nematoden, Copepoden, Polychaeten und Diatomeen.

\section{DISKUSSION}

Die Nahrung sedimentfressender Angehöriger der Makrofauna kann aus verschiedenen Komponenten bestehen: jungen Individuen der Makrofauna, Protozoen, Pilzen und Bakterien sowie aus partikulärer und gelöster organischer Substanz. Uber die relative Bedeutung dieser einzelnen Nahrungsbestandteile ist wenig bekannt. Daher sollte durch Darminhaltsuntersuchungen zunächst geprüft werden, ob Sipunculiden als reine Sedimentfresser Meiofauna aufzunehmen vermögen.

Die Nahrungsaufnahme geschieht bei allen Vertretern dieser Tiergruppe durch das 
Einziehen des Rüssels. Dabei bleiben Partikeln an den Papillen des Rüssels hängen und gelangen so in das Tier. Dieser Vorgang setzt vermutlich ein bestimmtes Größenverhältnis der Sipunculiden zu ihrer Nahrung voraus, so daß eine Selektion nach ihrer Größe anzunehmen war.

Elemente der Meiofauna konnten als Nahrung für die Sipunculiden festgestellt werden, und zwar in besonderem Maße die auch im Sediment dominierenden Nematoden und Crustaceen. Diese Angaben gelten besonders für Phascolosoma vulgare der Größenklassen II und III und für Phascolosoma elongatum der Größenklassen II und IV. Unterhalb dieser Größe nimmt die Häufigkeit der Nematoden bis auf wenige Exemplare ab. Crustaceen wurden in Phascolosoma vulgare unter $5,5 \mathrm{~cm}$ Länge kaum gefunden.

Diese Ergebnisse zeigen deutlich eine Selektion bestimmter Nahrungspartikeln, die sich vermutlich aus ihrer Größe ergibt. Die in Größenklasse I von Phascolosoma vulgare gefundenen Nematoden sind selten und um die Hälfte kleiner als die in Größenklasse III gefundenen Individuen (Tab. 3).

Nematoden winden sich stark bei jeder Störung und können dadurch wohl den Sipunculiden entkommen, oder sie entziehen sich der Aufnahme durch ihre Sperrigkeit. Bei den Crustaceen ergibt sich diese besonders durch die Extremitäten und die Antennen und bei vielen Nauplien durch ihre breitere Gestalt gegenüber den schlanken Harpacticiden. Auch die Flucht der auf dem Sediment lebenden beweglichen Organismen (besonders Crustaceen) dürfte eine Rolle für die Nahrungsselektion spielen. Bei Physcosoma granulatum ist die Bedeutung der Meiofauna als Nahrung offensichtlich geringer als bei den Phascolosoma-Arten, da die Individuen im Durchschnitt kleiner sind und die Möglichkeit, Meiofauna aufzunehmen, schon durch die Größenselektion verhindert wird.

Die Sipunculiden nehmen also eine Auswahl von Tieren der Meiofauna auf, so daß man von einer Nahrungsselektion sprechen kann. Diese ist allerdings nicht aktiv durch eine Leistung des fressenden Organismus bedingt, sondern sie ist als "passiv“ $\mathrm{zu}$ bezeichnen. Die für Nematoden und Crustaceen erwähnten Verhältnisse gelten ebenso für alle anderen Nahrungsorganismen. Größe und Beweglichkeit der Meiofauna sind also diejenigen Faktoren, die das Nahrungsangebot begrenzen.

Ein Vergleich der beiden untersuchten Gattungen Phascolosoma und Physcosoma als Vertreter verschiedener Biotope zeigt, wie die aufgenommene Nahrung abhängig ist vom umgebenden Sediment bzw. Nahrungsangebot und von dessen Beschaffenheit bezüglich Größe, Konsistenz und Mobilität. Physcosoma granulatum des Corralligène, die keinen direkten Kontakt zu einer Sedimentoberfläche haben und praktisch unbeweglich in den Kalkthalli sitzen, fressen meist tote, aber auch einige wenige größere und bewegliche Individuen (LAUbIER 1958). Sie „warten“ also auf die Nahrung, ein "passiver" Vorgang. Die Phascolosoma-Arten hingegen, als sich aktiv bewegende Bewohner der oberen $5 \mathrm{~cm}$ von feinen Sand- und Schlickböden, die ihre Nahrung von der Oberfläche bzw. den ersten $5 \mathrm{~mm}$ (nach Aufwühlen) entnehmen, stehen neben der Meiofauna ebenso der sedimentierte Detritus zur Verfügung. Meiofauna wird in größerem Maße aufgenommen als durch Pbyscosoma granulatum, da der Aktionsradius durch die Beweglichkeit größer ist.

Der Vergleich der beiden Arten zeigt, daß sich die Zusammensetzung der Nahrung 
in den sich entsprechenden Größenklassen gleicht. Physcosoma granulatum nimmt in etwas kleinerem Stadium bereits Crustaceen auf.

Die Oesophagusöffnung bildet bei den untersuchten Arten eine der individuellen Größe entsprechende Beengung, die ein „Filter“ für zu große und zu sperrige Formen darstellt.

Neben der Meiofauna werden auch andere Partikeln als Nahrung von den Sipunculiden aufgenommen. Die Schalen von Mollusken, Foraminiferen und die im Biotop in sehr großen Mengen vorkommenden Diatomeen werden regelmäßig im Darm der Sipunculiden gefunden, aber es ist nicht mehr sicher festzustellen, ob sie vor der Aufnahme verwertbare organische Substanz enthalten haben. Das gilt auch für Pflanzenreste, deren Cellulosestrukturen lange im Sediment erhalten bleiben, jedoch kaum als Nahrung genutzt werden dürften (GOERKE 1971b).

Bei den Sedimentuntersuchungen fanden sich als deutbare Partikeln auch Kotballen, die jedoch im Darm nicht mehr nachgewiesen werden konnten. Diese meist durch mucoidartige Substanzen zusammengehaltenen Partikeln werden vermutlich im Oesophagus zerstört. Die Rolle der Kotballen als Nahrung ist besonders durch ihren Gehalt an Bakterien gegeben (Frankenberg \& SMITH 1967), aber auch durch unverdaute organische Bestandteile (Conover 1966). Auch an den Sedimentpartikeln, besonders in der obersten Schicht, finden sich Bakterien in großer Zahl (z. B. MARE 1942, ZoBELL 1938, Raymont 1963, KRumbein 1971), die mitverdaut werden.

Für die Sipunculiden gilt also wie für Arenicola marina (NEwell 1965) und alle anderen Sedimentfresser, daß sie sowohl dem Ernährungstypus "microphagous feeders" als auch den "non-selective microphagous suspension feeders" (Klassifikation nach JoRGENSEN 1970) zuzuordnen sind. Außer den bereits erwähnten Nahrungskomponenten dürtte wenigstens in einigen Lebensräumen auch die gelöste organische Substanz als Nahrung mit von Bedeutung sein (SOUTHWARD \& SOUTHWARD 1971). Bei einer Wertung der Nahrungsanteile, die von Sedimentfressern aufgenommen und genutzt werden, müssen alle möglichen Komponenten berücksichtigt werden. Bisher ist allerdings keine Aussage über die relative Wertigkeit der einzelnen Anteile möglich, die auch in Abhängigkeit vom Nahrungsangebot - lokal und zeitlich - schwanken wird.

\section{ZUSAMMENFASSUNG}

1. Die Rolle der Meiofauna als Nahrung für Sipunculiden wurde an Phascolosoma vulgare (DE Blainville), Pbascolosoma elongatum (Keferstern) und Physcosoma granulatum (LEUCKART) geprüft. Die Sipunculiden konnten zur Beobachtung des Verhaltens bei der Nahrungsaufnahme im Laboratorium gehalten werden. Die Nahrung wurde nach Darminhaltsuntersuchungen an konservierten Exemplaren bestimmt.

2. Meiofaur:a wurde in allen Größenklassen der bearbeiteten Arten gefunden. In der Größenklasse I bei $1,0-2,4 \mathrm{~cm}$ und in der Größenklasse II bei 2,5-4,9 cm war die Anzahl der aufgenommenen Individuen auf Grund der Sperrigkeit der Crustaceen und der Beweglichkeit der Nematoden gering. Mit zunehmender Größe der Sipunculiden wird das Verhältnis zur Meiofauna als Nahrung günstiger. 
3. Bei einem Vergleich der im Darm der untersuchten Sipunculiden gefundenen Meiofauna mit derjenigen Meiofauna aus Sedimentproben wird deutlich, daß in Abhängigkeit von der Individuengröße nur Tiere bestimmter Größenklassen als Nahrung aufgenommen werden können.

4. Neben der Meiofauna umfaßt das Nahrungsangebot im Substrat auch Protozoen, Pilze und Bakterien sowie partikuläre abgestorbene und gelöste organische Substanzen. Mit der Größenzunahme der Sipunculiden verändert sich die relative Häufigkeit der aufgenommenen Bestandteile aus dem Nahrungsangebot. Eine quantitative Wertung der einzelnen Nahrungskomponenten konnte bisher noch nicht erfolgen.

Danksagungen. Herrn Dr. B. SweDMark von der Zoologischen Station Kristineberg/ Schweden möchte ich für die freundliche Aufnahme und Unterstützung während meines dortigen Aufenthaltes herzlich danken. Ebenso danke ich dem Zoologischen Staatsinstitut der Universität Hamburg für die Überlassung von Exkursionsmaterial des Jahres 1971.

\section{ZITIERTE LITERATUR}

Conover, R, J., 1966. Factors effecting the assimilation of organic matter by zooplankton and the question of superfluous feeding. Limnol. Oceanogr. 11, 339-345.

Frankenberg, D. \& Smtth, K. L., 1967. Coprophagy in marine animals. Limnol. Oceanogr. $12,443-450$.

GerLach, S. A., 1969. Ein Schema der Produktionsverhältnisse im Meer. Veröff. Inst. Meeresforsch. Bremerh. 11, 61-64.

- \& Schrage, M., 1969. Freilebende Nematoden als Nahrung der Sandgarnele Crangon crangon. Oecologica 2, 362-375.

Goerke, H., 1971a. Die Ernährungsweise der Nereis-Arten (Polychaeta, Nereidae) der deutschen Küsten. Veröff. Inst. Meeresforsch. Bremerh. 13, 1-50.

- 1971b. Nahrungsaufnahme, Nahrungsausnutzung und Wachstum von Nereis virens (Polychaeta, Nereidae). Veröff. Inst. Meeresforsch. Bremerh. 13, 51-78.

Hrman, L. H., 1959. The invertebrates: Smaller coelomates groups. McGraw-Hill, New York, 5, 1-783.

Jørgensen, C. B., 1966. Biology of suspension feeding. Pergamon Press, Oxford, 357 pp.

- 1970. Feeding mechanisms. In: Marine food chains. Ed. by J. H. Steele. Univ. of California Press, Berkely, Calif.

KrumbeIN, W. E.; 1971. Sediment microbiology and grain-size distribution as related to tidal movement, during the first mission of the West German Underwater Laboratory "Helgoland". Mar. Biol. 10, 101-112.

Ladider, L., 1958. Quelques données sur la biologie du Phascolosoma granulatum F. S. LeucKart 1828 (= Physcosoma granulatum [F. S. LeuckarT] auct.). Vie Milieu 9, 67-73.

- 1966. Le Corralligène des Albères. Annls. Inst. océanogr., Monaco, 43, 137-316.

McGinitie, G. \& McGnitre, N., 1949. Natural history of marine animals. McGraw-Hill, New York, 473 pp.

McIntyre, A. D., 1969. Ecology of marine meiobenthos. Biol. Rev, 44, 245-290.

MARE, M. F., 1942. A study of a marine community with special reference to the microorganisms. J. mar. biol. Ass. U.K. 25, 517-554.

Molander, A., 1928. Animal communities on the soft bottom areas in the Gullmars Fjord. (Kristinebergs Zoologiska Station 1877-1927.) SkrSer. K. svenska VetenskAkad. 1928 (2), $1-90$.

NEWELL, R, 1965. The role of detritus in the nutrition of two marine deposit feeders, the prosobrand Hydrobia ulvae and the bivalve Macoma baltbica. Proc. zool. Soc. Lond. 144, 25-45. 
Peebles, F. \& Fox, D., 1933. The structure, functions and general reactions of Dendrostoma zostericola. Bull. Scripps Inst. Oceanogr. tech. Ser. 3.

Raymont, E. G., 1963. Plankton and productivity in the oceans. Pergamon Press, Oxford, 660 pp.

Rhoads, D. C. \& Young, D. K., 1971. Animal-sediment relations in Cape Cod Bay, Massachusetts. 2. Reworking by Molpadia oolitbica (Holothuroidea). Mar. Biol. 11, 255-261.

SOUTHWARD, A. J. \& SOUTHWARD, E. C., 1972. Observations on the role of dissolved organic compounds in the nutrition of benthic invertebrates. II. Sarsia 48, 61-70.

Tétry, A., 1959. Les sipunculiens. Traité de zoologie. Ed. par P. P. Grassé. Masson, Paris, $5,785-854$.

ThreL, HJ., 1966. Quantitative Untersuchungen über die Meiofauna des Tiefseebodens (Vorläufiges Ergebnis der "Meteor"-Expedition in den Indischen Ocean). Veröff. Inst. Meeresforsch. Bremerh. (Sonderbd) 2, 131-147.

Zobell, C. E. \& Feltham, C. B., 1938. Bacteria as food for certain marine animals. J. mar. Res. 1, 312-317.

Anschrift der Autorin: Dipl.-Biol. Maria D. Watter

Institut für Hydrobiologie und Fischereiwissenschaft

2 Hamburg 50

Palmaille 55

Bundesrepublik Deutschland 